\title{
A new method for artificial chordae length "tuning" in mitral valve repair: Preliminary experience
}

Daniele Maselli, MD, Ruggero De Paulis, MD, Luca Weltert, MD, Andrea Salica, MD, Raffaele Scaffa, MD, Alessandro Bellisario, MD, and Antonio Mingiano, MD

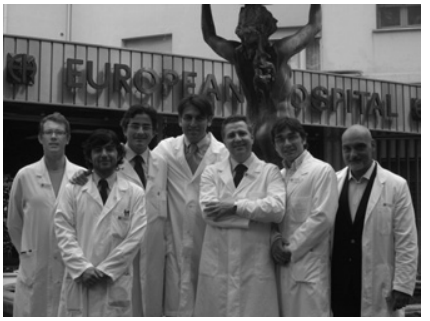

Drs Weltert, Scaffa, Maselli, De Paulis, Bellisario, Salica, and Mingiano (left to right)
From the Department of Cardiac Surgery, European Hospital, Rome, Italy.

Received for publication Feb 1, 2007; revisions received March 15, 2007; accepted for publication April 11, 2007.

Address for reprints: Daniele Maselli, MD, UO Cardiochirurgia, European Hospital, Via Portuense 700, 00149 Rome, Italy (E-mail: dmaselli@tiscali.it).

J Thorac Cardiovasc Surg 2007;134:454-9

$0022-5223 / \$ 32.00$

Copyright $\odot 2007$ by The American Association for Thoracic Surgery

doi:10.1016/j.jtcvs.2007.04.009
Objectives: Implanting expanded polytetrafluoroethylene neochordae is an established technique in treating complex mitral regurgitation. Difficulty in obtaining reliable preoperative and intraoperative measurements of an ideal neochordae length, as well as the unfeasibility of adjusting lengths once set in place with traditional techniques, led us to develop a system that allows rapid change of length after the evaluation of valve continence with hydrostatic tests.

Methods: The system consists of two components: a papillary component with arrest knots at constant intervals and a leaflet component with a reversible noose-lace to fix the loop to 1 of the knots on the papillary component. After implantation and coupling of the two components at a presumable optimal length, a prosthetic ring is sutured in place. Hydrostatic testing is then performed. Optimal chordae length can be obtained by releasing the noose-lace and sliding it over another fixing-knot. The adjustment can be performed as often as required without placing stress on the anatomic structures.

Results: Twenty patients underwent repair with this technique. No deaths or major adverse events occurred. All patients underwent echocardiography, both at discharge and 6 months after the operation. A total of 14 patients had no residual insufficiency, 5 patients had mild or trivial postoperative insufficiency without progression of regurgitation at the sixth month, and only 1 patient had mild insufficiency at discharge progressing to moderate insufficiency at the sixth month.

Conclusion: This new technique facilitates an otherwise complicated procedure. Short-term results are satisfactory, but further follow-up is required.

$\mathrm{M}$ itral valve chordae tendineae replacement with expanded polytetrafluoroethylene (ePTFE) sutures is an established technique for mitral valve repair. ${ }^{1}$ Artificial chordae have been successfully used in large series with excellent long-term results. ${ }^{2,3}$

Attempts to replace chordae, however, can be frustrated by difficulties encountered both in determining the optimal length of ePTFE sutures and in fixing them to valve leaflets and papillary muscles. Chordal length can be anticipated by echocardiography ${ }^{4}$ or by referring to healthy segments of the valve $e^{5}$; both methods suffer approximation because they lack a fixed and objective reference point. Experience and surgical skills are required to achieve optimal results. Moreover, expanded ePTFE sutures are slippery, and often knot tying can result in longer or shorter than planned neochordae. If intraoperative tests show that neochordae length is inappropriate, there is no other technical option but to replace them. ${ }^{6}$

We developed a configuration of polytetrafluoroethylene (Gore-Tex; WL Gore \& Associates, Inc, Flagstaff, Ariz) sutures by which the length of artificial chordae can be readjusted, as many times as required, without touching their papillary or leaflet anchoring, before fixing them in a definitive position. Our technique is described and preliminary experience is reported. 


\section{Abbreviations and Acronyms}

ePTFE $=$ expanded polytetrafluoroethylene

\section{Materials and Methods Population}

From October 1, 2004, to November 1, 2006, 20 patients (eight male, mean age $60.2 \pm 9.1$ years) underwent mitral valve repair procedure with artificial chordae implantation after acquiring informed consent. Only patients with no need for concomitant procedures were included in the study. The patients' characteristics, intraoperative findings, details regarding techniques used, and results are illustrated in Table 1.

\section{Surgical Technique}

Our method for mitral chordae replacement is based on reversible coupling of two ePTFE components: the leaflet component and the papillary component.

The leaflet component is achieved with a CV-5 polytetrafluoroethylene (Gore-Tex) suture. A circular loop is obtained at the middle of the suture by tying it around a Hegar dilator with a diameter of $13 \mathrm{~mm}$. When the loop is flattened, its length equals half the circumference. Given a circumference of approximately 4 $\mathrm{cm}$ for a circle with a diameter of $13 \mathrm{~mm}$, the final length of the loop will be approximately $2 \mathrm{~cm}$ (Figure 1). The papillary component is obtained by cutting a CV-4 polytetrafluoroethylene (Gore-Tex) suture in two halves. After this, 5 double surgeon's knots, at a fixed distance of $2 \mathrm{~mm}$, are placed at the needleless tip of each CV-4 semisuture. To facilitate correct positioning of the knots, the needleless tip of the suture is anchored on a drape, knots are made with the help of forceps and a needle holder, and then, given the slippery properties of the ePTFE fabric, slid into definitive position by inserting the tip of the needle or a nerve hook in the knot itself. Two CV-4 half sutures with knots are needed to realize the papillary component of each neochorda (Figure 1). Chordae components are achieved during preparation for cardiopulmonary bypass.

After assessment of the mitral valve lesions, the papillary component is set in place first by fixing 2 semisutures to a papillary head and tying on the sutures so that the papillary head is "sandwiched" between 2 ePTFE pledgets to reduce trauma (Figure 1). Sutures are left inside the ventricle. Two CV-5 loops are then fixed on the desired leaflet, 2 to $3 \mathrm{~mm}$ apart from the leaflet's edge, passing the needle from the atrial to the ventricular side and leaving knots on the ventricular aspect of the leaflet. A single ePTFE pledget is interposed on the atrial side (Figure 1).

To achieve reversible coupling of the leaflet component with the papillary component of the neochorda, a loop that can be tightened and loosened as many times as required is placed in the leaflet component with the help of forceps and a curved instrument

\section{TABLE 1. Population characteristics}

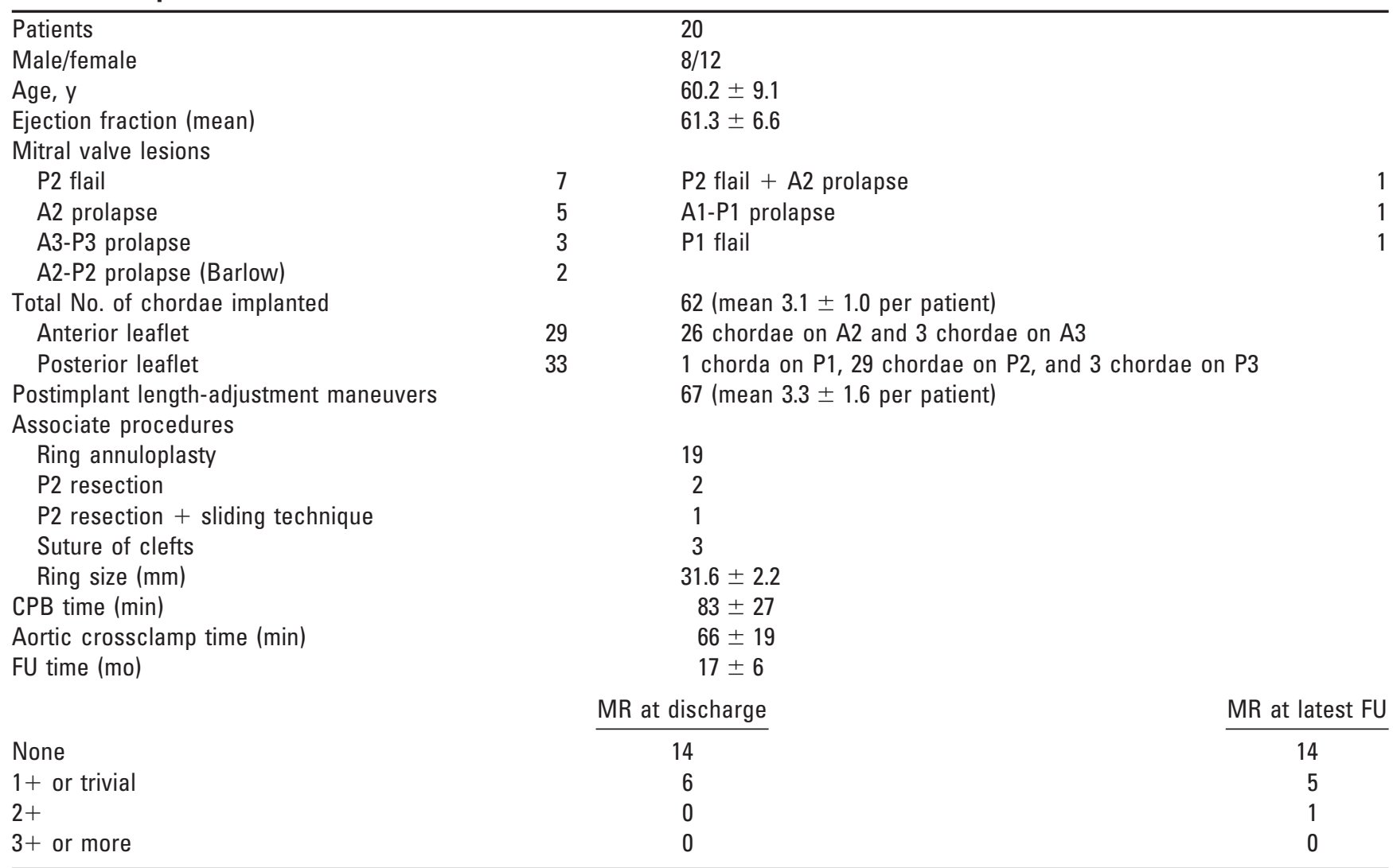

$C P B$, Cardiopulmonary bypass; $F U$, follow-up; $M R$, mitral regurgitation. See text for description of the techniques. 

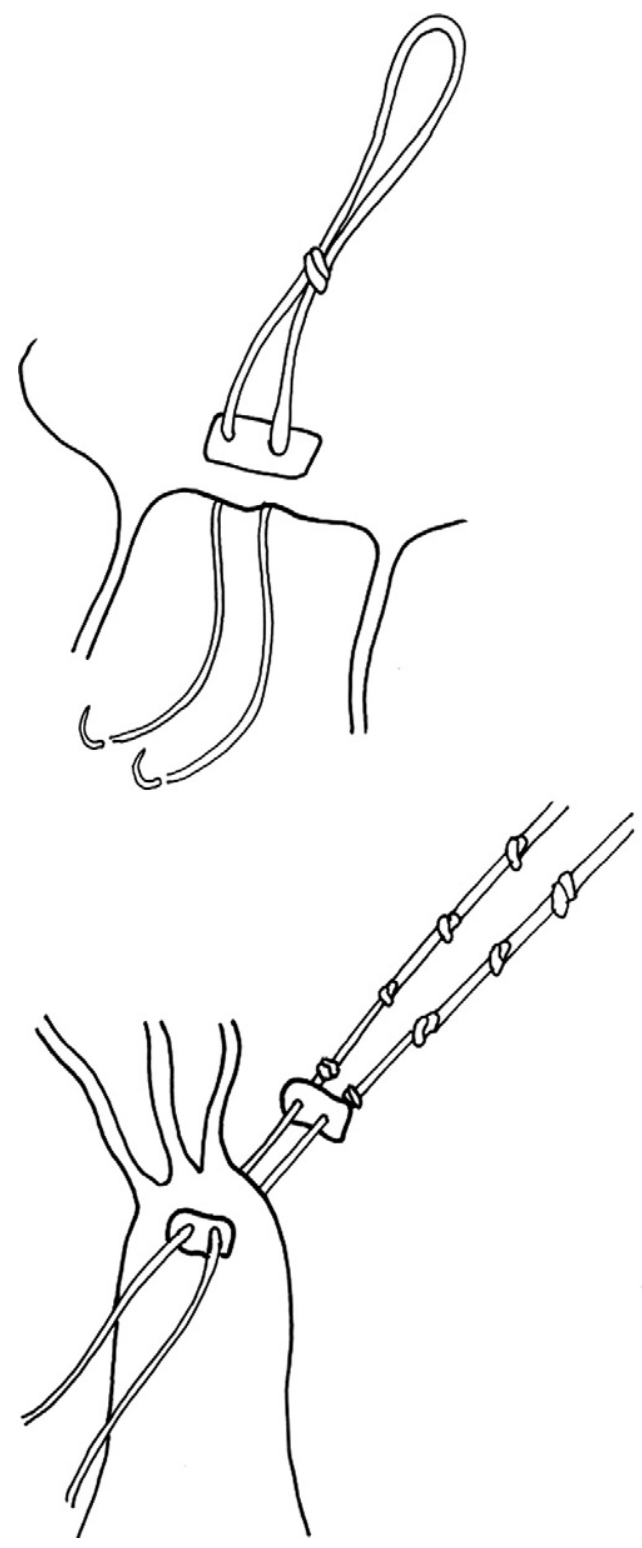

Figure 1. The papillary component (down) is fixed at the papillary muscle head. ePTFE pledgets are used to reduce trauma. The leaflet component (up) is fixed at the desired portion of the mitral valve leaflet, leaving knots on the ventricular side.

(Figure 2). The papillary component is then passed inside the loop, and the loop is tightened; the loop has to fall in the gap between two knots. The chordal length is fixed by closing the loop under the selected reference knot of the papillary component (Figure 3 ). The maneuver is repeated for the other chordae. To shorten or elongate the neochorda without touching its papillary or leaflet anchoring, the loop is released (to release the joint between the 2 components, the tip of the discarded CV-5 suture needle is inserted inside the loop and the leaflet component is opened), slid under a reference knot respectively closer or farther from the papillary muscle tip, and tightened again.

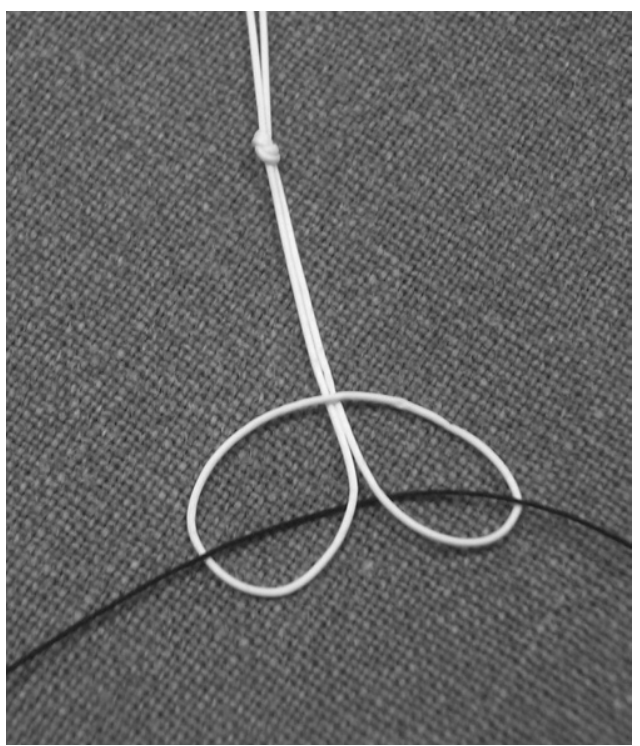

Figure 2. A loop is created on the implanted leaflet component by pulling the free end with forceps and folding it by $\mathbf{1 8 0}$ degrees; this maneuver creates 2 small rings. The papillary component is then passed inside both rings, and coupling is attained by closing the loop and pulling the 2 components in opposite directions. The course of the papillary component inside the loop (black line).

Competence of the mitral valve is checked by saline infusion into the left ventricle. Even if the lengths of all the chordae have to be changed, that can be easily achieved at this time. When a satisfactory result is achieved, the loop is closed by firmly pulling the two components in opposite directions, and the excess length of the papillary component is trimmed.

To evaluate the static properties of the new configuration and its failure load in comparison with the standard procedure, the artificial chordae were loaded in an uniaxial machine (Instron 5542, Norwood, Mass; load cell of 50N) and strains were calculated by an optical extensometer: The images were recorded by a camera (Nikon Coolpix 4500, Tokyo, Japan) during the tests and analyzed with a specific Matlab code (The MathWorks Inc, Natick, Mass), referring to ink-colored markers positioned along the sutures.

\section{Follow-up}

A systematic mitral valve analysis was carried out intraoperatively and at 6 months follow-up by transesophageal echocardiography using a Sonos 5005 echocardiograph (Hewlett Packard, Andover, Mass) equipped with a Philips T6210 omniplane transesophageal echocardiography probe (Philips, Bothell, Wash). Valve lesions were categorized according to Carpentier's classification. ${ }^{7}$ Grading of residual mitral regurgitation is reported according to indications of the American Society for Echocardiography. ${ }^{8}$

\section{Results}

Our technique was successful in all patients. All patients survived. No complications were reported in the in-hospital 


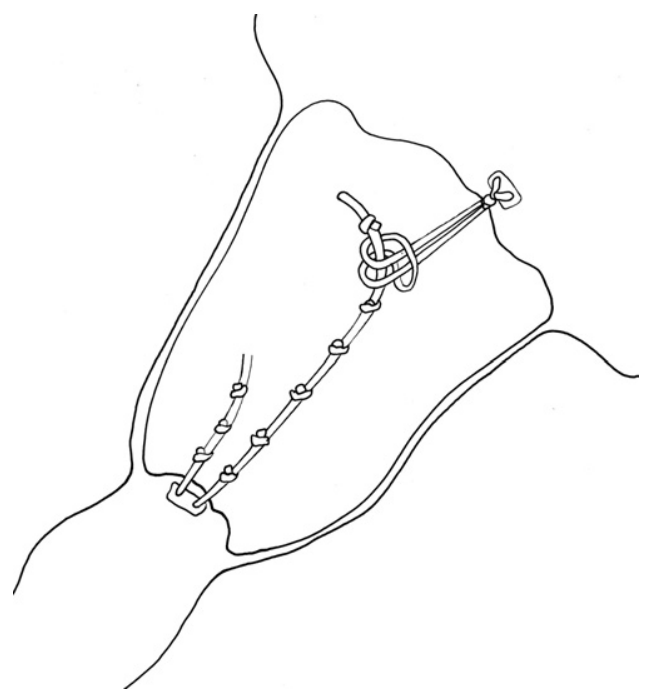

Figure 3. The papillary component is passed inside the leaflet component, and the loop is tightened just below the desired knot. The loop can be loosened and repositioned as many time as needed to achieve a perfect valve competence. Once the result is considered satisfactory, the exceeding portion of the ventricular component is cut and discarded.

postoperative course except for 3 patients (15\%) who experienced paroxysmal atrial fibrillation treated by amiodarone infusion. Patients in sinus rhythm received only antiplatelet drugs; anticoagulation was reserved for patients experiencing atrial fibrillation. No neurologic events were reported. No major events were reported at 6 months follow-up. Regurgitation grade was stable except for a single patient who experienced a worsening of mitral regurgitation from $1+$ to $2+$, not apparently related to chordal dehiscence. In this patient, flail P1 and P2 segments of the posterior leaflet and massive annular calcification were observed at operation and treated by positioning two leaflet components: one on P1 and one on P2. An annuloplasty ring was not implanted because of massive annular calcifications. No instances of neochordae rupture were documented at follow-up.

\section{Discussion}

Solutions proposed to adjust the length of ePTFE chordae during mitral valve repair surgery can be grouped in two major categories.

In the first group of techniques, artificial chordae are first sutured on the papillary muscle tip and then on the mitral valve leaflets; knot tying is deferred after open heart mitral valve competence tests have shown an effective coaptation.

A method to adjust chordal length was described by Revuelta and colleagues ${ }^{9}$ in a sheep model. A doublearmed, pledget-supported ePTFE suture was passed through the anterior papillary muscle and then woven into the mitral leaflet from the edge toward the annulus where it was brought out and passed through a second pledget. Valve competence was obtained by pulling on the ePTFE suture while filling the left ventricle with blood through the apical vent. Suture length was fixed by tying at that time. Kasegawa and colleagues ${ }^{6}$ reported the use of small tourniquets to adjust the length of artificial chordae, during open mitral valve competence testing, before definitive knot tying. In the method described by Adams and coworkers, ${ }^{10}$ a doublearmed 4-0 ePTFE suture is passed as a simple suture into the head of the papillary muscle and left aside while leaflet reconstruction is performed. After ring annuloplasty, both arms of the suture are passed twice through the prolapsing portion of the leaflet and tied starting with a surgeon knot after valve competence has been achieved with saline injections into the left ventricle.

These methods are obviously independent of echocardiography or intraoperative measurements and are free from errors that can be induced by using "healthy segments" of the valve as reference points. The final results can be significantly affected by knot tying and eventual knot sliding.

To set the length of neochordae and to avoid the effect of knot tying on the suture's length, Sarsan ${ }^{11}$ proposed placing a temporary edge-to-edge suture that is removed only after knot tying, between the normal and prolapsing facing portions of the mitral valve. Rankin and colleagues ${ }^{12}$ recently proposed a technique to attain adjustable chordae: Sutures are first passed through the head of the papillary muscle previously reinforced by "sandwiching" it between two pledgets and then, after positioning the annuloplasty ring, through the leaflet. A slipknot is attained and temporarily fixed with a clip. If competence tests fail, the clip is removed and knot slipped by $1-\mathrm{cm}$ increments until a satisfactory competence is achieved. Eight more knots are tied on the clip that is subsequently removed. With this technique, the chordal length is fully adjustable and not affected by knot tying.

A second group of techniques relies on the creation of premeasured ePTFE loops or on devices that measure chordal length and avoid knot migration at the same time.

Von Oppel and $\mathrm{Mohr}^{13}$ described a method to attain premeasured chordae by referring to nonprolapsing segments of the valve. Premeasured loops are fixed on the papillary muscle and then by a second suture on the desired segment of the leaflet. Matsui and coworkers ${ }^{14}$ introduced a device to measure chordae of normal valve segments and to stop knots during knot tying. These methods eliminate the potentially deleterious effect of knot tying. The final length of artificial chordae, however, is entirely dependent on preoperative or intraoperative measurements. 
TABLE 2. Tensile strength of CV4 size tested with and without knots at the two different test rates

\begin{tabular}{lcr}
\hline \multicolumn{3}{c}{ Maximum load (N) } \\
\hline Suture & $\mathbf{2 ~} \mathbf{~ m m} / \mathbf{m i n}$ & $\mathbf{1 0 ~} \mathbf{~ m} / \mathbf{m i n}$ \\
\hline Simple & $10.9 \pm 0.3$ & $13.6 \pm 0.6$ \\
Knotted & $10.5 \pm 0.6$ & $14 \pm 0.7$ \\
\hline
\end{tabular}

All the techniques described share a theoretic limit: If the final length is incorrect, artificial chordae have to be removed and replaced. This can result in weakening of papillary muscles and mitral valve leaflets and can determine a significant prolongation of myocardial ischemic time. If inappropriate chordal length becomes apparent after an annuloplasty ring has been positioned, it can be impractical to implant new chordae with the ring in place.

Our technique eliminates the need to premeasure chordae by echocardiography and to refer to "healthy" segments of the valve. The surgeon simply needs to connect the two components to leaflet and papillary muscle to attain coupling. Because the papillary and leaflet components are fixed before coupling, the effect of knot-tying maneuvers on the final chordae length is eliminated. Artificial chordae length can be adjusted, without repositioning sutures on mitral valve leaflets or papillary muscles, as often as required. Length adjustment can be attained even if a prosthetic ring is already in place. Because adjustment steps are located only $2 \mathrm{~mm}$ apart, our technique results in effective fine-tuning and customization of chordal length.

In our preliminary experience, 67 chordal lengthadjustment maneuvers were performed on 62 implanted chordae. That is, all chordae in question had an incorrect length at the first coupling or the technique led to multiple attempts, occasionally resulting in overcorrection (22 of these maneuvers were made to return to the previous length). This demonstrates the relative utility of the system.

A series of steps were followed, consisting primarily of implanting the papillary components, followed by placing annular sutures for ring positioning. Leaflet reconstruction is then performed if required, followed by the implantation of the leaflet components. Coupling, tests, and adjustment maneuvers are performed only after positioning the annuloplasty ring. This sequence, postponing chordal set-up after ring positioning (which almost always affects the quality and extent of mitral leaflet coaptation), is significantly time-saving.

One potential limitation of the technique is that knots made on the papillary component to stop the closed loop of the leaflet component could slide under tension, and this could result in chordal elongation or opening of the system. Mechanical properties of the new suture configuration have been thoroughly investigated by us. ${ }^{15}$ Tensile tests revealed
TABLE 3. Tensile strength of different configuration tested

\begin{tabular}{ll}
\hline \multicolumn{2}{c}{ Maximum load (N) } \\
\hline Configuration & $\mathbf{2 ~} \mathbf{~ m m} / \mathbf{m i n}$ \\
\hline Standard & $15.4 \pm 6.8$ \\
New & $15.5 \pm 1.5$ \\
\hline
\end{tabular}

that knots do not significantly affect the strength of the suture; in fact, simple and knotted sutures reached similar ultimate loads (Table 2). Adjustable-length chordae exhibit a kind of perfect plastic behavior with rather high strains at rupture; their static properties are comparable to the standard technique (Table 3).

A second limitation is the possibility that the loop on the leaflet component could open spontaneously in an eventual depowered situation in which chordae are not under tension. However, in the early 1960s while studying chordae tendineae tension, Salisbury and colleagues ${ }^{16}$ demonstrated that the diastolic tension on chordae tendineae, in the nonfailing heart with the pericardium closed, is never less than $12 \mathrm{~g}$. This means that human chordae tendineae do have a relative resting phase but are always under tension. This is reflected in our clinical experience in which no instances of failure or opening of the system could be documented at follow-up.

With our system, a continuous adjustment of chordal length is not possible. Length adjustment depends on the fixed distance of $2 \mathrm{~mm}$ between knots on the papillary component. This is a potential limitation in cases in which a fine-tuning of chordal length is desired. In our initial clinical experience, however, results were encouraging.

\section{Conclusions}

This new technique to determine the proper length of mitral neochordae has the advantage of easily correcting for small errors when judging the optimal length of the neochordae. A simple modification in the point of coupling of the two chordae components precludes the need to repeat the whole surgical procedure. Although further follow-up is required, early clinical results are promising. The possibility of having premanufactured artificial chordae as described would greatly facilitate their use.

\section{References}

1. David TE. Artificial chordae. Semin Thorac Cardiovasc Surg. 2004; 16:161-8.

2. Chiappini B, Sanchez A, Noirhomme P, Verhelst R, Rubay J, Poncelet A, et al. Replacement of chordae tendineae with polytetrafluoroethylene (PTFE) sutures in mitral valve repair: early and long-term results. Heart Valve Dis. 2006;15:657-63.

3. Kasegawa H, Shimokawa T, Shibazaki I, Hayashi H, Koyanagi T, Ida T. Mitral valve repair for anterior leaflet prolapse with expanded polytetrafluoroethylene sutures. Ann Thorac Surg. 2006;81:1625-31.

4. Fabricius AM, Walther T, Falk V, Mohr FW. Three-dimensional echocardiography for planning of mitral valve surgery: current applicability? Ann Thorac Surg. 2004;78:575-85. 
5. Zussa C, Polesel E, Da Col U, Galloni M, Valfrè C. Seven-year experience with chordal replacement with expanded polytetrafluoroethylene in floppy mitral valve. J Thorac Cardiovasc Surg. 1994;108:37-41.

6. Kasegawa H, Kamata S, Hirata S, Kobayashi N, Mannouji E, Ida T, et al. Simple method for determining proper length of artificial chordae in mitral valve repair. Ann Thorac Surg. 1994;57:237-9.

7. Carpentier A. Cardiac valve surgery: the French correction. $J$ Thorac Cardiovasc Surg. 1983;86:323-37.

8. Zoghbi WA, Enriquez-Sarano M, Foster E, Grayburn PA, Kraft CD, Levine RA, et al. Recommendations for evaluation of the severity of native valvular regurgitation with two-dimensional and Doppler echocardiography. J Am Soc Echocardiogr. 2003;16:777-802.

9. Revuelta JM, Garcia-Rinaldi R, Gaite L, Val F, Garijo F. Generation of chordae tendineae with polytetrafluoroethylene stents. J Thorac Cardiovasc Surg. 1989;97:98-103.

10. Adams DH, Kadner A, Chen RH. Artificial mitral valve chordae replacement made simple. Ann Thorac Surg. 2001;71:1377-9.
11. Sarsan MAI. Simplified technique for determining the length of artificial chordae in mitral valve repair. Ann Thorac Surg. 2002; 73:1659-60.

12. Rankin JS, Orozco RE, Rodgers TL, Alfery DD, Glower DD. "Adjustable" artificial chordal replacement for repair of mitral valve prolapse. Ann Thorac Surg. 2006;81:1526-8.

13. Von Oppel UO, Mohr F. Chordal replacement for both minimally invasive and conventional mitral valve surgery using premeasured Gore-Tex loops. Ann Thorac Surg. 2000;70:2166-8.

14. Matsui Y, Fukada Y, Naito Y, Sasaki S, Yasuda K. A new device for ensuring the correct length of artificial chordae in mitral valvuloplasty. Ann Thorac Surg. 2005;79:1064-5.

15. Celi S, Di Puccio F, Bajona P, Maselli D. Mechanical testing of a new suture configuration for the replacement of chordae tendineae. $J$ Biomech. 2006;39:S619.

16. Salisbury PF, Cross CE, Rieben PA. Chorda tendinea tension. Am J Physiol. 1963;205:385-92. 